DOI: https://doi.org/10.46296/gt.v4i8edesp.0027

\title{
TRASTORNO DEPRESIVO PERSISTENTE Y TRASTORNOS DE ANSIEDAD GENERALIZADA PROVENIENTE DE UN ESTRÉS POST TRAUMÁTICO CRÓNICO: PRESENTACIÓN DE UN CASO CLÍNICO
}

\section{MIXED ANXIETY AND DEPRESSION DISORDER FROM CHRONIC POST TRAUMATIC STRESS: PRESENTATION OF A CLINICAL CASE}

\author{
Arredondo-Aldana Karina ${ }^{1}$; Mera-Posligua Mauro Julio ${ }^{2}$; \\ Ponce-Alencastro Jhon Alexander ${ }^{3}$ \\ ${ }^{1}$ Estudiante de la Maestría Académica con Trayectoria de Investigación en Psicología, Mención \\ Psicoterapia. Instituto de Posgrado de la Universidad Técnica de Manabí, UTM. Portoviejo, \\ Ecuador. Correo: karyarredondoaldana@gmail.com. ORCID ID: https://orcid.org/0000-0002- \\ 9874-6657 \\ ${ }^{2}$ Estudiante de la Maestría Académica con Trayectoria de Investigación en Psicología, Mención \\ Psicoterapia. Instituto de Posgrado de la Universidad Técnica de Manabí, UTM. Portoviejo, \\ Ecuador. Correo: mjmera95@gmail.com. ORCID ID: https://orcid.org/0000-0001-9560-6395 \\ ${ }^{3}$ Estudiante de la Maestría Académica con Trayectoria de Investigación en Psicología, Mención \\ Psicoterapia. Instituto de Posgrado de la Universidad Técnica de Manabí, UTM. Portoviejo, \\ Ecuador. Correo: jhon.ponce@utm.edu.ec. ORCID ID: https://orcid.org/0000-0002-3666-7865
}

\section{Resumen}

El trastorno depresivo persistente, es un trastorno afectivo crónico que permanece al menos por dos años en adultos y un año en niños y adolescentes. Los pacientes con este diagnóstico se pueden mostrar pesimistas, regularmente negativos, con bajo o nada sentido del humor, muy pasivos, retraídos, muy críticos hacia sí mismos y también hacia los otros. Los pacientes con este trastorno tienen probabilidades de presentar trastornos de ansiedad generalizada, el cual se caracteriza por presentar preocupación excesiva y continua por asuntos que son desproporcionados a lo que realmente se vive, se percibe constantemente un futuro amenazante, esto es difícil de controlar por la persona e interfiriere en su rutina diaria. Muchas veces estos trastornos pueden ser provenientes de otros enfermedades o trastornos, como, por ejemplo, del estrés post traumático que se desarrolla a raíz de haber vivido o presenciado un acontecimiento traumático como lo es una agresión sexual, estos pacientes a lo largo del tiempo no se sienten mejor, sino más bien estresados o asustados después de que la situación traumática haya concluido, en el siguiente caso clínico se presume que la sintomatología ansiosa y depresiva fue desarrollada a raíz de un trastorno de estrés post traumático cronificado.

Palabras clave: trastorno depresivo persistente, ansiedad generalizada, estrés post traumático, terapia cognitivo-conductual.

\begin{abstract}
Persistent depressive disorder is a chronic affective disorder that lasts for at least two years in adults and one year in children and adolescents. Patients with this diagnosis can be pessimistic, regularly negative, with little or no sense of humor, very passive, withdrawn, very critical of themselves and also of others. Patients with this disorder are likely to present generalized anxiety disorders, which is characterized by excessive and continuous concern for issues that are disproportionate to what is actually experienced, a threatening future is constantly perceived, this is difficult to control by the person and interferes with your daily routine. Many times these
\end{abstract}

Información del manuscrito:

Fecha de recepción: 13 de julio de 2021.

Fecha de aceptación: 27 de septiembre de 2021.

Fecha de publicación: 12 de noviembre de 2021. 
disorders can be derived from other diseases or disorders, such as, for example, post traumatic stress that develops as a result of having lived or witnessed a traumatic event such as a sexual assault, these patients over time do not they feel better but rather stressed or scared after the traumatic situation has concluded, in the following clinical case it is presumed that the anxious and depressive symptoms were developed as a result of chronic post-traumatic stress.

Keywords: persistent depressive disorder, generalized anxiety, post traumatic stress disorder, cognitive behavioral therapy.

\section{Introducción}

La Asociación Americana de Psiquiatría, DSM-5, (2014), menciona que el trastorno depresivo persistente se caracteriza por un estado de ánimo deprimido durante la mayor parte del día y debe estar presente durante el periodo de dos años; en los niños y adolescentes, el estado de ánimo puede ser irritable y la duración ha de ser como mínimo un año, durante la depresión se deben presentar dos 0 más síntomas, tales como, poco apetito o sobrealimentación, insomnio o hipersomnia, fatiga, baja autoestima, falta de concentración, dificultad para tomar decisiones $\mathrm{y} / \mathrm{o}$ sentimientos de desesperanza. Los pacientes con este trastorno tienen mayores posibilidades de padecer comorbilidad de trastornos de ansiedad.

El trastorno de ansiedad generalizada (TAG) se define como una ansiedad y preocupación excesiva que debe estar presente al menos un mínimo de seis meses, en relación con diversos sucesos 0 actividades $\mathrm{Al}$ individuo le es difícil controlar la preocupación (Asociación Americana de Psiquiatría, 2014), esto se manifiesta con una sensación de ansiedad no placentera, difusa, acompañada de molestias físicas como opresión retroesternal, taquicardia, sudoración, náuseas, diarrea y/o mareos (Carvajal, A., 2016).

Los pacientes con trastorno de ansiedad generalizada muestran variaciones en su funcionalidad a nivel ocupacional, ya que se quejan frecuentemente de problemas cognitivos y falta de confianza (Langarita, R.; Gracia P., 2019). Estudios han descrito que las mujeres presentan el doble de probabilidad que los hombres de experimentar este trastorno 
(Asociación Americana de

Psiquiatría, 2014).

Autores como Arango, C. y Rincón, $\mathrm{H}$. (2016) mencionan que dentro de los factores de riesgo para presentar TAG, se encuentran los genéticos, fisiológicos, temperamentales y ambientales. Dentro de los factores genéticos y fisiológicos se refieren a las alteraciones genéticas que interfieren en la regulación de neurotransmisores. Dentro de los factores temperamentales destaca la inhibición afectiva, el neuroticismo y tendencia a la evitación del daño. En los factores ambientales tienden a ser personas en estados de soledad que cuentan con escaso contacto social, generando estrés psicosocial y ocasionando cambios a nivel de los receptores de glucocorticoides, disfunción del eje hipotálamohipofiso-adrenal y aumento en la liberación de citocinas.

Bermúdez, L., (2020) menciona que el trastorno de estrés postraumático (TEPT) es definido como una reacción emocional penetrante ante un suceso traumático, mostrando alteración del sentido, la conciencia o la conducta, que amenaza el bienestar o la vida del individuo.

Gualpa, G., (2020) menciona que, en 1889 Oppenheim, describió el TEPT como "neurosis traumática" ligado casi siempre con enfrentamientos agresivos, catástrofes naturales, agresiones sexuales y con frecuencia también a accidentes de tránsito graves o con riesgo vital. Éste afecta a alrededor de 5.2 millones de adultos, siendo más frecuente en mujeres que en hombres, no hay correspondencia con la edad y existe la certeza de que la susceptibilidad a un estrés postraumático suele ser genético y a menudo suele acompañarse con signos de ansiedad y depresión en diferentes grados.

La clasificación de los trastornos mentales y del comportamiento, CIE10, (2000) refiere que las características típicas de este trastorno son episodios repetidos de volver a vivenciar el trauma a través de reviviscencias o sueños, además suele presentarse una sensación de "entumecimiento" y embotamiento emocional, de despego de los demás, de falta de capacidad de respuesta al medio, de anhedonia y 
de evitar actividades y situaciones evocadoras del trauma. La persona le teme a las situaciones que sugieren 0 recuerdan al trauma evitándolas, además se exterioriza un estado de hiperactividad vegetativa con hipervigilancia, se incrementa la reacción de sobresalto e insomnio. Los síntomas además se acompañan de ansiedad y depresión, así como también se pueden presentar ideaciones suicidas. En una pequeña proporción de los enfermos, el trastorno puede tener durante muchos años un curso crónico y evolución hacia una trasformación persistente de la personalidad.

El presente caso se tratará mediante el modelo Cognitivo - Conductual, del cual Barrera, N. y Reyes, J., (2020) refieren que este se fundamenta en la unión de los enfoque cognitivo y conductual, los cuales son primordiales en el estudio del comportamiento humano y los factores intervinientes. Este enfoque reflexiona sobre cómo la raza humana procesa y aprende la información, además de cómo reconoce al contexto de acuerdo a sus experiencias, conocimientos, pensamientos, genética y etapa evolutiva. Por lo tanto, este modelo estudia el cómo mediante lo visto o experimentado se crean pensamientos e ideas que conllevan a una respuesta favorable o no para el contexto que se vive. Es decir, primero se observa, luego se procesa la información, para continuar aprendiéndola y finalmente ejecutado lo asimilado.

En lo referente a la eficacia, diversos estudios evidencian que dicha terapia es efectiva en dificultades de depresión, trastorno de ansiedad generalizada, de dolor crónico, trastorno por estrés postraumático, trastorno obsesivo compulsivo, entre otros (Alcántara, 2016; Ferro-García et al., 2017; Losada et al., 2015; Luciano et al., 2016)

Dentro de las técnicas de este enfoque se ocuparán las siguientes técnicas:

- Psicoeducación: surge a finales del siglo XX, como proceso en el que un profesional de salud brinda información sobre el origen, evolución y tratamiento de un padecimiento al paciente y a la familia, en caso de ser necesario (Cuevas, J. y Moreno, N., 2017). Por otro lado, 
Ramírez, E. y Vizcaíno, A. (2020) refieren que la psicoeducación es un proceso educativo que se encamina a estimular los recursos psicológicos en los individuos por lo que puede promover el bienestar emocional. Se ha elegido esta técnica, para enseñarle a la paciente sobre sus diagnósticos y cómo manejar las diferentes situaciones que se le puedan presentar a raíz de sus problemáticas, por lo que combina la educación sobre la problemática a enfrentar con la entrega de estrategias de resolución de problemas.

- Relajación muscular progresiva de Jacobson: según Ruíz, M., et al, (2017) esta es una técnica sencilla y factible de aprender y pueden ser utilizadas por la población en general. Los supuestos básicos de esta técnica son intentar ser una técnica fisiológica que se centra en la relajación de la musculatura, por otro lado, este entrenamiento se debe apoyar en la discriminación de los signos de tensión en los diversos músculos del cuerpo a través de adiestramientos sistémicos de tensión- relajación. De igual manera la relajación de los músculos esqueléticos-motores conlleva instintivamente a la relajación del sistema autonómico-vegetativo y con esto a la relajación del sistema nervioso central. La relajación a nivel fisiológico ocasiona efectos a la par de la relajación emocional. Mediante esta técnica se desea enseñar a la paciente a que centre su atención en las sensaciones que pueda tener de tensión y distención de los 4 grupos musculares para alcanzar una desactivación fisiológica, a fin de poder relajarse después de hablar sobre los abusos sexuales que sufrió.

- Reestructuración cognitiva: esta técnica está encaminada a enseñar al paciente a que intervenga en sus propios pensamientos negativos de índole automáticos, a que identifique la similitud entre cognición, emoción y conducta, 
también a que identifique evidencias tanto a favor como en contra de sus cogniciones distorsionadas, cambiar los pensamientos irracionales por otros más racionales y, por último, a que aprender a identificar y modificar las creencias no realistas (Mendoza, I., 2020). Se utiliza esta técnica para reestructurar las preocupaciones sobre el futuro y el trastorno de la paciente, corregir sus creencias irracionales de que todos los hombres se acercarán para abusar de ella, de la misma manera se utiliza esta técnica para que ella aprenda a eliminar los pensamientos negativos con respecto a los abusos sexuales que tuvo en la infancia y principios de la adolescencia.

- Entrenamiento en solución de problemas: Badanes, M. (2017) menciona que el objetivo que tiene este entrenamiento es optimizar la capacidad de afrontamiento de los pacientes ante estresores; los objetivos específicos de esta técnica son acrecentar la orientación positiva hacia las dificultades y por ende que la orientación negativa hacia ellos se reduzca, animar la solución planeada de problemas, que se reste la evitación que tiene el paciente hacia los problemas, reducir la solución impulsiva de los mismos. Por el contrario, también existen obstáculos que se presentan a la hora de alcanzar los objetivos, tales como son: los sentimientos de impotencia, la sobrecarga cognitiva, la pobre regulación emocional, el procesamiento cognitivo sesgado, y la planificación ineficaz de las habilidades de solución ante los problemas. Con esto se desea explicar a la paciente las siguientes fases de la técnica: orientación hacia el problema, definición del problema, generación de soluciones alternativas, toma de decisión, ejecución y verificación de estas.

- Técnica de reducción de estrés: esta técnica pretende enseñar a la paciente aquellas variables sobre las que las personas pueden incidir, tales como mantener una dieta equilibrada, descansar lo necesario, realizar 
ejercicios, planificación y organización del tiempo. (Carro, C., Sanz, R., 2015). Se utiliza esta técnica con la paciente para poder conseguir que reduzca y prevenga la ansiedad y el estrés que presenta.

- Planificación y gestión del tiempo: técnica que se enfoca en que la paciente aprenda a planificar y gestionar el tiempo de manera adecuada. (Carro, C., Sanz, R., 2015). Se le enseña esto a la paciente con el fin de que pueda descansar, no estar tan preocupada por sus actividades, no sufrir sobresaltos, olvidos importantes, entre otras.

A continuación, se presenta el proceso de evaluación e intervención terapéutica de la paciente.

\section{Metodología}

\subsection{Caso clínico}

Paciente de 20 años, de género femenino con tendencia homosexual, soltera, estudiante universitaria, hija de padres casados, ocupa el tercer lugar dentro de los tres hijos que tiene el matrimonio y además tiene 2 hermanos por parte de padre. Acude a consulta derivada por psiquiatría quien la diagnosticó con trastorno mixto de ansiedad y depresión.

Hija deseada, no se utilizan medicamentos durante el 1er mes de embarazo, no refiere traumas ni golpes, el embarazo se desarrolló sin complicaciones y el parto fue mediante cesárea y sin dificultades. A los 6 años sufre una caída de cabeza en un pasamanos, producto de esto se desmayó y se rompió la nariz. En la escuela tenía buenas notas y buenas relaciones con los compañeros. Creció sufriendo de abuso sexual por parte de un tío, de los dos hermanos mayores y de un primo mayor, todo esto sucedió durante 6 años.

Considera que su adolescencia fue buena hasta los 14 años donde comenzó con una "depresión", debido a que se ilusionó con un chico y cuando eso terminó se "deprimió mucho". A los 15 años tuvo problemas alimenticios pero los padres nunca se enteraron, un año después les cuenta a los padres del abuso sexual que había sufrido en su infancia y parte de su adolescencia, 
ellos la apoyaron y la llevaron a una psicóloga, además tuvo un intento de suicidio mediante ingestión de medicamentos.

Actualmente tiene una pareja de género femenino, desde hace tres años, con quien se lleva bastante bien y tiene relaciones sexuales sin problemas. Antes de esta relación tuvo algunos novios en el colegio, con un solo chico duró un año y llegó a tener relaciones sexuales con él, pero no fue placentero, ella trataba de complacer, pero ella siente que él no la complacía.

Mantiene una buena relación con los padres, pero no les tiene confianza, no tienen conocimiento de su orientación sexual ni tampoco de que se considera agnóstica. Con los hermanos la relación es buena, con el hermano mayor no se ven mucho porque ya no vive con ellos, con el hermano del al medio que es quien vive con los padres y ella tiene bastante confianza. Tiene pocas amigas, pero son muy unidas y no tiene amigos hombres.

Consume alcohol casualmente y aunque no pierde la conciencia si se embriaga, durante 2 años y medio fumó marihuana de manera intermitente y durante 4 años se autolesionó

de

manera

ininterrumpida.

Como antecedente psicopatológicos familiares refiere que las dos abuelas padecen Alzheimer, padre con diabetes, tías con cáncer de seno y un tío con cáncer de piel.

Su problema actual comienza desde hace 7 años cuando se autolesionaba, luego a los 17 tuvo un ataque de pánico, en la universidad comenzó a consumir marihuana y pasaba mucho rato "ida", siempre buscaba la manera de "desaparecer", refiere que siempre ha sentido que su vida no tiene un propósito, por lo cual ha querido suicidarse, sólo una vez lo intentó tomando pastillas, pero las vomitó y los padres no supieron de eso, de ahí para adelante sólo ha tenido recurrentes ideaciones suicidas. Desde hace algunas semanas refiere manifestar sintomatología depresiva, ya que sólo salía de la cama para recibir clases, comer y saludar a mamá, duerme bastante de tarde, es más activa de noche, de día se siente adormitada y sin ganas de hacer nada. Siente ansiedad del futuro, de saber si se mejorará o siempre estará así, suele tener recuerdos recurrentes en forma de 
flash back sobre los abusos del tío principalmente. En la actualidad no tiene contacto con el principal autor de los abusos, no va a la casa de la abuela que es donde él vive, cuando lo ve en eventos familiares ella se pone tensa y ansiosa. Refiere tener desconfianza y recelo con todos los hombres, incluso con el papá siempre intenta mantener la distancia. Cuando va por la calle y va cerca de hombres se pone ansiosa porque piensa que la van a tocar, si le dicen cosas estos hombres ella se pone muy mal después. No usa ropa corta ni con escote si es que sale sola.

Este problema le afecta de manera directa a la paciente ya que está presentando problemas de comunicación y de relación con la familia, problemas para relacionarse con las personas del sexo masculino, desconfianza, recelo aislamiento social y familiar, además presenta como síntomas secundarios fatiga, taquicardias, problemas en la concentración, problemas de memoria, problemas para dormir, baja autoestima, dificultad para tomar decisiones, tensión muscular, irritabilidad e inquietud.

\subsection{Exámen de funciones mentales}

Aspecto un poco descuidado, colaboradora ante la entrevista, vigil, con conciencia de la situación y de la enfermedad, orientada auto y alopsíquicamente, euproséxica, ideas sobrevaloradas, ideas suicidas, pensamiento rígido y de contenido obsesivo, sin alteraciones motrices, eulálica, tono de voz monótona, distimia displacentera y tenacidad afectiva, anhedonia, sensopercepciones sin alteración, juicio desviado.

\subsection{Baterías psicológicas}

- Escala de ansiedad de Hamilton: Escala que consta de 14 ítems, 13 de ellos hacen mención a signos y síntomas ansiosos, el último está reservado para evaluar el comportamiento del paciente durante la entrevista. La persona evaluada debe ir puntuando la intensidad en la que se exteriorizan los síntomas ansiosos basándose en una escala Likert de 5 valores categoriales, estos están basados en: ausente, leve, moderado, grave y muy grave. (López, M., 2018). Se utilizó esta escala para 
valorar el nivel de ansiedad que presenta la paciente y su resultado fue de 54 puntos, lo que quiere decir, que presenta un trastorno de ansiedad, tal como se piensa.

- Escala de depresión de Hamilton: Escobar, A. y Peñaloza, M., (2017) mencionan que este es un instrumento psicométrico que se utiliza para medir el nivel de depresión de una persona, siendo una de las pruebas psicométricas más usadas en temas de investigación. Esta prueba se utilizó para valorar el nivel de depresión que presenta la paciente, obteniendo un total de 32 puntos, lo que significa que su grado de depresión es severa.

- Escala de gravedad de síntomas del trastorno de estrés postraumático (EGS-R): es una escala heteroaplicada, la cual tiene una estructura tipo likert que va de 0 a 3 según la repetición e intensidad de los síntomas. Consta de 21 ítems que van en correlación con los criterios diagnósticos del DSM-5:de ellos 5 referencian los síntomas de reexperimentación, con un rango de 0 a 15 puntos, 3 corresponden a los de evitación conductual/cognitiva, que van en un rango de 0 a 9 puntos, otros 7 dirigidos a alteraciones cognitivas y estado de ánimo negativo, que van en un rango de 0 a 21 puntos, y 6 referidos a síntomas de aumento de la activación y reactividad psicofisiológica, con un rango de 0 a 18 puntos. Se considera un síntoma presente cuando se puntúa, al menos, con dos puntos en el ítem correspondiente. El rango de la escala global oscila de 0 a 63 puntos. Se utiliza esta escala con la paciente para evaluar la gravedad de los síntomas que presenta, obteniendo los siguientes resultados, 15/15 puntos en lo que se refiere a reexperimentación, lo que quiere decir que es un síntoma que está presente; 9/9 puntos en evitación conductual/cognitiva, lo que significa que es un síntoma presente en la paciente; 19/21 puntos en la alteración cognitivo/ estado de ánimo negativo, lo que refiere que esto se encuentra presente en la paciente; 17/18 puntos en aumento de activación y reactividad psicofisiológica lo 
que refiere que el síntoma si está presente en ella; $60 / 63$ en lo que se refiere a la gravedad del TEPT, lo que refleja que es grave en la paciente este trastorno; así mismo se refleja en esta escala que la paciente presenta más de dos síntomas disociativos; y que hay un alto grado de afectación del suceso (18/18 puntos).

\subsection{Diagnóstico presuntivo}

F34.1 Trastorno depresivo persistente grave de inicio temprano
F41.1 Trastorno de ansiedad generalizada

F43.10 Trastorno por estrés posttraumático crónico

\subsection{Evolución}

El tratamiento de la paciente se realizará en 21 sesiones, de esta manera la paciente acuerda con el psicoterapeuta asistir a sesiones cada 15 días con una duración de una hora en cada encuentro. Las sesiones se llevarán a cabo de la siguiente manera:

Tabla 1. Sesiones y técnicas de tratamiento.

\begin{tabular}{|c|c|c|}
\hline SESION (ES) & $\begin{array}{l}\text { TÉCNICAS DE } \\
\text { TRATAMIENTO }\end{array}$ & OBJETIVO \\
\hline 1,2 y 3 & $\begin{array}{l}\text { Entrevista inicial, } \\
\text { evaluación clínica }\end{array}$ & $\begin{array}{l}\text { Esto se realiza con la paciente para conocer sobre } \\
\text { su historia de vida y su problemática, para luego } \\
\text { llegar a un diagnóstico y planificar su plan } \\
\text { terapéutico. Acá se realiza un listado sobre las } \\
\text { conductas problemas de la paciente. }\end{array}$ \\
\hline 4 y 5 & $\begin{array}{l}\text { Psicoeducación y } \\
\text { presentación de } \\
\text { videos sobre } \\
\text { trastornos } \\
\text { depresivo } \\
\text { persistente, } \\
\text { ansiedad y estrés } \\
\text { post traumático }\end{array}$ & $\begin{array}{l}\text { Esto se realiza para entregarle información científica } \\
\text { a la paciente sobre trastorno depresivo persistente, } \\
\text { ansiedad y TEPT, para que de esta manera } \\
\text { adquiera un conocimiento para poder enfrentar de } \\
\text { mejor manera las diversas situaciones que se le } \\
\text { presenten. }\end{array}$ \\
\hline 6 & $\begin{array}{ll}\text { Pautas } & \text { de } \\
\text { reducción } & \text { de } \\
\text { estrés } & \end{array}$ & $\begin{array}{l}\text { Se le enseñan a la paciente una serie de pautas } \\
\text { para reducir los niveles de estrés que le provoquen, } \\
\text { por ejemplo, las situaciones cuando debe salir de la } \\
\text { casa y estar rodeada de hombres en diferentes } \\
\text { escenarios. Acá se le enseña a la paciente sobre la } \\
\text { importancia de llevar una dieta equilibrada, de } \\
\text { dormir las horas necesarias, el realizar ejercicios de } \\
\text { manera moderada y también de lo importante que } \\
\text { es planificar con tiempo sus actividades de rutina. }\end{array}$ \\
\hline
\end{tabular}




\begin{tabular}{|c|c|c|}
\hline $6-10$ & $\begin{array}{ll}\text { Técnica } & \text { de } \\
\text { desactivación } & \\
\text { fisiológica } & \\
\text { (relajación } & \\
\text { muscular } & \\
\text { progresiva } & \text { de } \\
\text { Jacobson) } & \end{array}$ & $\begin{array}{l}\text { Esto se realiza para que la paciente aprenda a } \\
\text { reducir la activación fisiológica que le provocan sus } \\
\text { crisis de ansiedad, a su vez para que después de } \\
\text { cada sesión que se hable sobre el tema de los } \\
\text { abusos sexuales pueda relajar el cuerpo de la } \\
\text { tensión que le provoca el hablar sobre esta temática. } \\
\text { Se utiliza la técnica abreviada de } 4 \text { grupos } \\
\text { musculares, en la primera fase se aplica la relajación } \\
\text { de brazos, en la segunda el área facial, nuca, } \\
\text { hombros y espalda, en la tercera pecho, estómago } \\
\text { y vientre y en la cuarta fase caderas, muslos y } \\
\text { pantorrilla, y para terminar se realiza una relajación } \\
\text { de cuerpo completo. }\end{array}$ \\
\hline $7-18$ & $\begin{array}{l}\text { Reestructuración } \\
\text { Cognitiva }\end{array}$ & $\begin{array}{l}\text { Esto se aplica para mejorar el estado de ánimo } \\
\text { negativo, preocupaciones sobre el futuro y } \\
\text { pensamientos sobre sus posibles recaídas de } \\
\text { ánimo, así como también para ir mejorando su } \\
\text { estado de ánimo deprimido y los recuerdos con } \\
\text { pensamientos negativos que pueda tener sobre los } \\
\text { abusos sexuales recibidos en su infancia e inicio de } \\
\text { su adolescencia. Se le enseña a la paciente a } \\
\text { identificar los errores de pensamientos que comete } \\
\text { y se le envía como tarea para la casa un } \\
\text { autorregistro sobre situación, pensamiento, error de } \\
\text { pensamiento cometido, emoción y pensamiento } \\
\text { alternativo a dichos errores. En las siguientes } \\
\text { sesiones se procede a revisar la tarea y a discutir los } \\
\text { pensamientos y creencias irracionales o } \\
\text { distorsiones cognitivas que se presentan de manera } \\
\text { más habitual. Se utilizarán preguntas tales como: } \\
\text { ¿qué evidencia hay sobre este pensamiento?, ¿eso } \\
\text { es así para todo el mundo?, ¿qué evidencias tiene } \\
\text { en contra de su pensamiento?, ¿consigue algo } \\
\text { pensando así?, ¿hay otra forma de interpretar las } \\
\text { cosas? }\end{array}$ \\
\hline $17-18$ & $\begin{array}{l}\text { Entrenamiento en } \\
\text { solución de } \\
\text { problemas y toma } \\
\text { de decisiones }\end{array}$ & $\begin{array}{l}\text { Esto para aprender a solucionar problemas y tomar } \\
\text { nuevas decisiones de una manera adecuada antes } \\
\text { las diversas situaciones que le toque enfrentar. Aquí } \\
\text { se le plantea a la paciente una pregunta ¿qué } \\
\text { problemas tienes? Luego entre la psicóloga y la } \\
\text { paciente se realiza una lista con todas las posibles } \\
\text { soluciones al problema, luego se procede a analizar } \\
\text { las posibles soluciones y decidir cuales o cuál } \\
\text { utilizará para luego enfrentar la situación que le está } \\
\text { dificultando. }\end{array}$ \\
\hline 19 & $\begin{array}{l}\text { Planificación y } \\
\text { gestión del tiempo }\end{array}$ & $\begin{array}{l}\text { Se realiza con la paciente un horario para que } \\
\text { aprenda a planificar y gestionar su tiempo de mejor } \\
\text { manera y pueda mantenerse en actividades } \\
\text { agradables y reducir de esta manera los } \\
\text { pensamientos negativos y reducir sus niveles de } \\
\text { ansiedad }\end{array}$ \\
\hline
\end{tabular}




\begin{tabular}{|l|l|}
$\begin{array}{l}\text { Prevención de } \\
\text { recaídas }\end{array}$ & $\begin{array}{l}\text { Se hace en conjunto con la paciente un repaso por } \\
\text { todas las estrategias aprendidas en sesión y una } \\
\text { autoevaluación post - tratamiento }\end{array}$
\end{tabular}

\subsection{Objetivos psicoterapéuticos}

- Detectar creencias pensamientos irracionales junto a la paciente, dentro de la problemática que padece.

- Desarrollar habilidades para el correcto manejo de sus emociones

- Aumentar la autoeficacia y el control de los síntomas ansiosos y depresivos para que retome sus actividades cotidianas.

- Desarrollar habilidades para corregir los pensamientos irracionales sobre su problemática.

- Psicoeducar a la paciente sobre la distimia, el trastorno de ansiedad generalizada y el TEPT para mejorar el reconocimiento de ideas irracionales

- Descubrir los pensamientos, sentimientos y emociones que le provoca el acontecimiento traumático

- Promover el reconocimiento como victima

- Permitir el acercamiento normal hacia el sexo masculino, el cual le ha provocado malestar a partir de la reexperimentación dentro de la problemática.

\section{Resultados y discusión}

En concordancia con lo expuesto la paciente cuenta con un doble diagnóstico como resultado de un estrés postraumático crónico, esto concuerda con los criterios diagnósticos del DSM-5 para trastorno depresivo persistente $y$ ansiedad generalizada, lo que no quiere decir que el TEPT no esté presente, sino que los dos trastornos mencionados están prevaleciendo por sobre el TEPT en la actualidad en la vida de la paciente.

Se diagnostica con trastorno depresivo persistente ya que la paciente ha presentado el rasgo característico de este trastorno como es un estado deprimido que está presente la mayor parte del día y la mayor parte de los días durante más de dos años (DSM-5, 2014), además de otros criterios diagnósticos mencionados en este manual; se 
diagnostica ansiedad generalizada porque cumple el criterio diagnóstico principal mencionado en el DSM-5, (2014) que es una ansiedad y preocupación descomunales acerca de una serie de sucesos 0 actividades, conjuntamente la duración, intensidad o frecuencia es exagerada al impacto real del suceso anticipado, así también cumple con otros criterios tales como menciona Carvajal, A., (2016) que son impresión de ansiedad que no es placentera para la paciente, ésta es difusa, se acompaña de molestias físicas como opresión en el pecho, taquicardias, sudoración, náuseas, diarrea y/o mareos.

El principal alcance de la TCC es que disminuye los síntomas del TEPT, la ansiedad, la depresión, y puede mejorar la calidad del sueño (National Center for PTSD, 2015). No obstante, en la terapia con la paciente se presenta una limitación como es hablar sobre el trauma en el comienzo de las sesiones. Sin embargo, Alcántara, 2016; FerroGarcía et al., 2017; Losada et al., 2015; refieren que esta terapia es eficaz en dificultades de depresión, trastorno de ansiedad generalizada, de dolor crónico, trastorno por estrés postraumático, trastorno obsesivo compulsivo, entre otros.

\section{Conclusiones}

El presente caso se trata de una paciente de sexo femenino que presenta un trastorno depresivo persistente y un trastorno de ansiedad generalizada, en sesiones se logra comprender que estos diagnósticos provienen de un estrés post traumático crónico desarrollado en su niñez por los diversos abusos sexual por parte de familiares hacia ella, este TEPT no fue nunca tratado.

Se espera que con el tratamiento planificado, en base al modelo cognitivo - conductual, la paciente reduzca sus niveles de ansiedad, reconozca sus pensamientos e ideas irracionales y los modificarlos a pensamientos positivos y racionales, además superar el cuadro de estrés post traumático, para reducir el foco de su depresión por las experiencias vividas, disminuir los niveles de ansiedad con respecto a su futuro y superar el hecho de tener que compartir espacios abiertos 0 cerrados con hombres, producto del temor de que quieran abusar de ella. 
Se espera que la paciente pueda llevar un estilo de vida funcional, afrontar y superar lo que le sucedió en su niñez, no recaer constantemente en depresión y disminuir sus ideaciones suicidas.

\section{Bibliografía}

Alcántara, M. (2016). Intervenciones positivas y psicopatología centrada en depresión. [Tesis de fin de grado), Universidad de Jaén, Andalucía, España], http://tauja.ujaen.es/jspui/bitst ream/10953.1/3401/1/Alcntar a_Castro_Miriam_TFG_Psico loga.pdf

Arango, C, Rincón, H. (2018). Trastorno depresivo, trastorno de ansiedad y dolor crónico: múltiples manifestaciones de un núcleo fisiopatológico y clínico común. Revista colombiana de psiquiatría, 47 , 46-55, http://doi.org/10.1016/j.rcp.20 16.10.007

Asociación Americana de Psiquiatría. (2014). Manual Diagnóstico y Estadístico de los Trastornos Mentales DSM-5. (5 ${ }^{\mathrm{a}}$ Edición). España: Editorial Panamericana

Badena, M. (2017). Terapia de solución de problemas (TSP) en personas afectadas por la crisis económica, [tesis máster; Universidad Jaumb-I], https://core.ac.uk/download/p df/153569836.pdf

Barrera, N., Reyes, J. (2020). Revisión literaria del trastorno de ansiedad un abordaje desde los enfoques de la psicología. Revisión documentaria derivado del diplomado- Habilidades para la intervención clínica, https://repository.ucc.edu.co/ bitstream/20.500.12494/1671 3/3/2020_trastorno_ansiedad .pdf

Bermúdez, L., Barrantes, M., Bonilla, G. (2020). Trastorno por estrés postrauma. Revista Médica Sinergia, 9, https://doi.org/10.31434/rms.v $5 i 9.568$

Carro, C., Sanz, R. (2015). Intervención cognitivo conductual en un caso de distimia con componentes de ansiedad de evaluación: caso clínico. Revista de casos clínicos en salud mental, 1, 43-59,

file:///C:/Users/Usuario/Downl oads/Dialnet-

IntervencionCognitivoConduc tualEnUnCasoDeDistimiaC5912890.pdf

Carvajal, A., Flores, M., Marin, S., Morales C. (2016). Los trastornos de ansiedad durante la transición a la menopausia. Perinatología y reproducción humana, 30, 3945 , 
http://dx.doi.org/10.1016/j.rpr h.2016.03.003

Cuevas, j., Moreno, N. (2017). Psicoeducación: intervención de enfermería para el cuidado de la familia en su rol de cuidadora. Enfermería universitaria, 14, 207-218, https://doi.org/10.1016/j.reu.2 017.06 .003

Echeburúa E., Amor, P., Sarasua, B., Zubizarreta, I., Holgado, F., Muñoz, J. (2016). Escala de Gravedad de Síntomas Revisada (EGS-R) del Trastorno de Estrés Postraumático según el DSM5: propiedades psicométricas. Terapia psicológica, 34, 111128 , https://scielo.conicyt.cl/pdf/ter psicol/v34n2/art04.pdf

Escobar, A., Peñaloza, M. (20172018). Relación del ambiente familiar, consumo de sustancias, y calidad de vida, con ansiedad y depresión, en adolescentes de 12 a 17 años, que asisten a centros de acogida y adicciones, en quito, Cotacachi, Pusuquí, y Guayaquil, periodo noviembre 2017 a julio 2018. [Trabajo fin especialidad, Pontificia universidad Católica del Ecuador], http://repositorio.puce.edu.ec/ bitstream/handle/22000/1555 8/TRABAJO\%20DE\%20TITU LACI\%C3\%93N.pdf?sequenc $\mathrm{e}=1$ \&isAllowed $=\mathrm{y}$
Ferro-García, R., Ascanio, L., \& Valero, L. (2017). Integrando la terapia de aceptación y compromiso con la terapia de interacción padres-hijos en un niño con trastorno negativista desafiante. Revista de Psicología Clínica con Niños y Adolescentes, 4(1), 33-40, http://www.revistapcna.com/si tes/default/files/16-03.pdf

Gualpa, G., Choca, E., Basantes, D. (2020). Trastorno de estrés Postraumático; experiencias en Ecuador en la población de Riobamba. Polo de conocimiento, 5, 663-654, https://polodelconocimiento.c om/ojs/index.php/es/article/vi ew/1302/2318\#

Langarita, R., Gracias, P. (2019). Neuropsicología del trastorno de ansiedad generalizada: revisión sistemática. Revista de Neurología/formación online, 69, 59-67, DOI: https://doi.org/10.33588/rn.69 02.2018371

López, M., (2018). Intervenciones positivas y psicopatología centrada en depresión. [Título de grado, Universidad de Jaén, Andalucía, España]. https://repositorio.pucesa.edu .ec/bitstream/123456789/217 5/1/76597.pdf

Losada, A., Márquez, M., Romero, R., López, J., Fernández, V., \& Nogales, C. (2015). Atendiendo a las variadas problemáticas de los cuidadores familiares de 
personas con demencia: aportaciones de la terapia cognitivo-conductual y de la terapia de aceptación y compromiso. Clínica y Salud, 26(1), 41-48. doi:1016/j.clysa.2015.02.001

Mendoza,I. (2020). Terapia cognitivo conductual: Actualidad, tecnología, [tesis licenciatura, Universidad peruana Cayetano Heredia], https://repositorio.upch.edu.p e/bitstream/handle/20.500.12 866/8907/Terapia_MendozaF ernandez_Ingrid.pdf?sequenc $\mathrm{e}=1$ \&isAllowed=y

National Center for PTSD. (2015). Tratamientos eficaces para el TEPT: Considerar la terapia cognitivo-conductal (TCC) como tratamiento de primera elección. National Center for post traumatic stressdisorder, https://www.ptsd.va.gov/spani sh/docs/EffectiveTreatmentP TSD_Physicians_SP.pdf

Ramírez, E,. Vizcaíno, A. (2020). La psicoeducación y su alcance para el desarrollo de adultos en el contexto laboral. Didasc@lia: Didáctica y Educación, XI,107-123, http://revistas.ult.edu.cu/index .php/didascalia/article/view/11 07/1110

Ruiz, M., Reyes, A., Carrasco M. (2017). Estrés y ansiedad. Técnica de relajación muscular progresiva de Jacobson, Revista Electrónica de Portales Medicos.com, https://www.revista-

portalesmedicos.com/revistamedica/estres-ansiedadtecnica-relajacion-muscularprogresiva-jacobson/

World Health Organization. (2000). Guía de bolsillo de la clasificación CIE-10: clasificación de los trastornos mentales y del comportamiento. Madrid: Editorial Médica Panamericana. https://apps.who.int/iris/handl e/10665/42326 